\title{
Coupling of First Principles and Machine Learning for High Throughput Calculation of Evaporation Fields
}

\author{
Joaquin Peralta ${ }^{1}$, Scott Broderick $^{2}$, Claudia Loyola $^{1}$, and Krishna Rajan ${ }^{2}$ \\ 1. Departamento de Ciencias Físicas, Facultad de Ciencias Exactas, Universidad Andrés Bello, Santiago \\ 8370136, Chile \\ 2. Department of Materials Design and Innovation, University at Buffalo, Buffalo, NY 14260, USA
}

The objective of this work is to understand the field evaporation process through a high throughput data driven approach. We link atomic probe tomography (APT) experiment, first principles computation, and informatics to (i) model the degradation mechanism under extreme fields, (ii) develop new modeling approach for field based on experimental calibration, and (iii) develop high throughput models for predicting evaporation field as function of chemistry, governing experimental design strategy. A challenge which we address is the ion deviation from the ideal positions due to our limited understanding of ion trajectory near the tip immediately after evaporation. While the ion trajectory can be accurately modeled away from the tip based on geometrical factors, the trajectory and evaporation field are impacted by neighborhood chemical arrangement [1], laser heating, and electronic effects [2]. This represents a significant challenge which we have previously addressed through density functional theory (DFT) calculations, where the relationship between evaporation field, materials chemistry, and relative bonding were correlated (Figure 1).

This work develops the materials design triumvarate of experiment, computation and informatics. The experiment-computation link results in atomic scale measurement with electronic scale degradation mechanisms, the experiment- informatics link leads to high throughput prediction of APT parameters, and finally the computation-informatics link defines the relationship between chemistry and property. Our objective is to understand the mechanism associated with the change in evaporation fields (ie. required applied voltage) by performing DFT calculations to measure evaporation field as a function of chemistry and structure. We connect the field evaporation (Fe) with chemistry - ie. scaled descriptors associated with chemistry such as radii, electronegativity, charges, bonding, etc. From DFT / experiment, we determine the evaporation field for both single element systems and semi-conductors, with additional parameters associated with instrumental parameters when available (such as from the EPOS file). We use kernel principal component analysis to describe correlations by decomposing the data into a matrix which allows us to visualize data with non-linear physics incorporated into the model. The initial results from the informatics analysis provides a high throughput model between chemistry and evaporation field (Figure 2), which can be applied for improved reconstruction and material selection. The integration of the seperate experiment, computation and informatics thrusts contribute to a full design platform linking physical understanding with empirical measurement.

References:

[1] J. Peralta, S.R. Broderick, and K. Rajan. Ultramicroscopy 132 (2013) p. 143.

[2] C. Loyola, J. Peralta, S.R. Broderick, K. Rajan, J. Vacuum Sci. Tech. A 34 (2016) p. 061404.

[3] This work is supported by Proyecto FONDECYT Iniciación 11130501 and NSF DIBBs Award Number ACI-1640867 

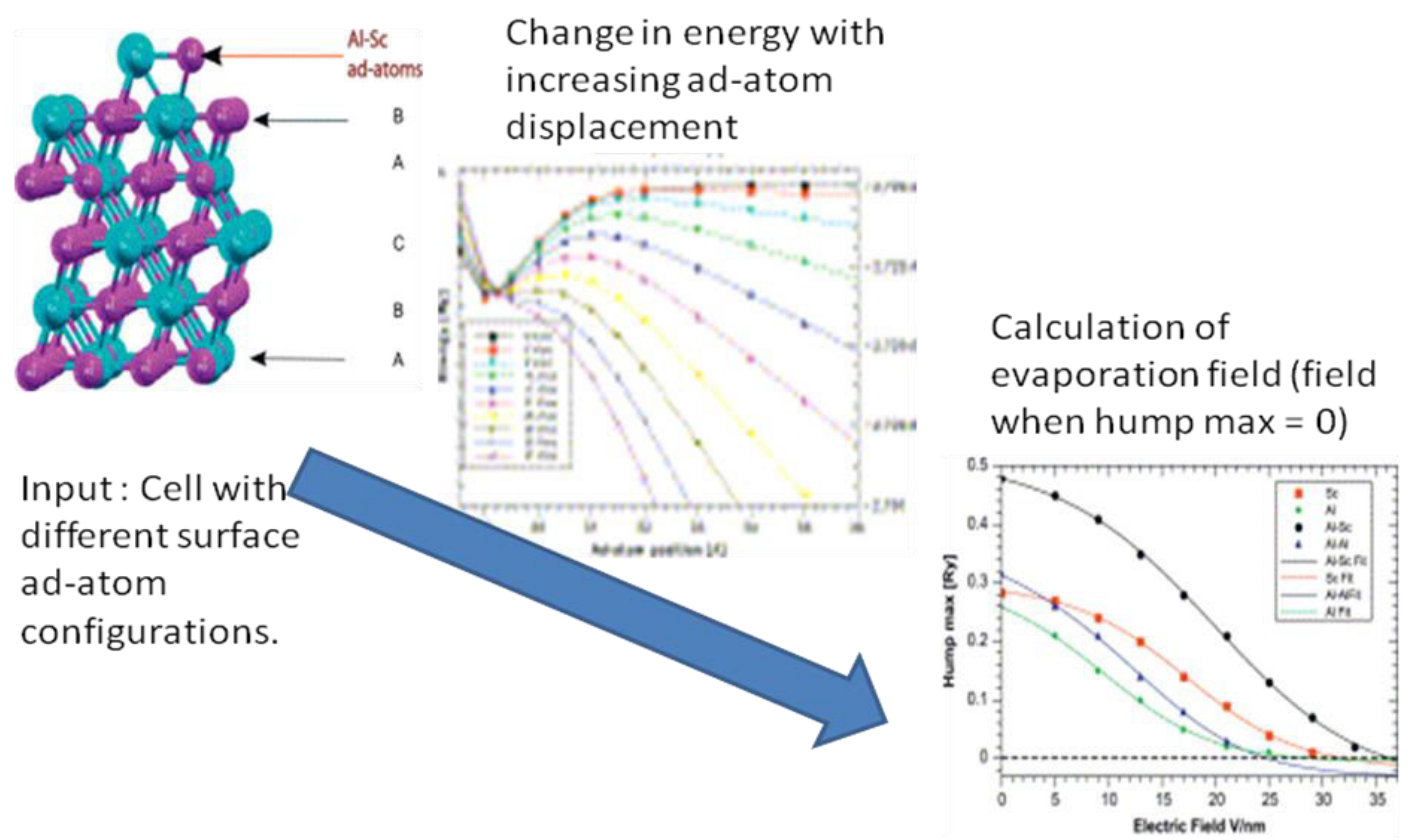

Figure 1. Developed DFT approach for defining evaporation field as a function of surface chemistry and evaporation physics.

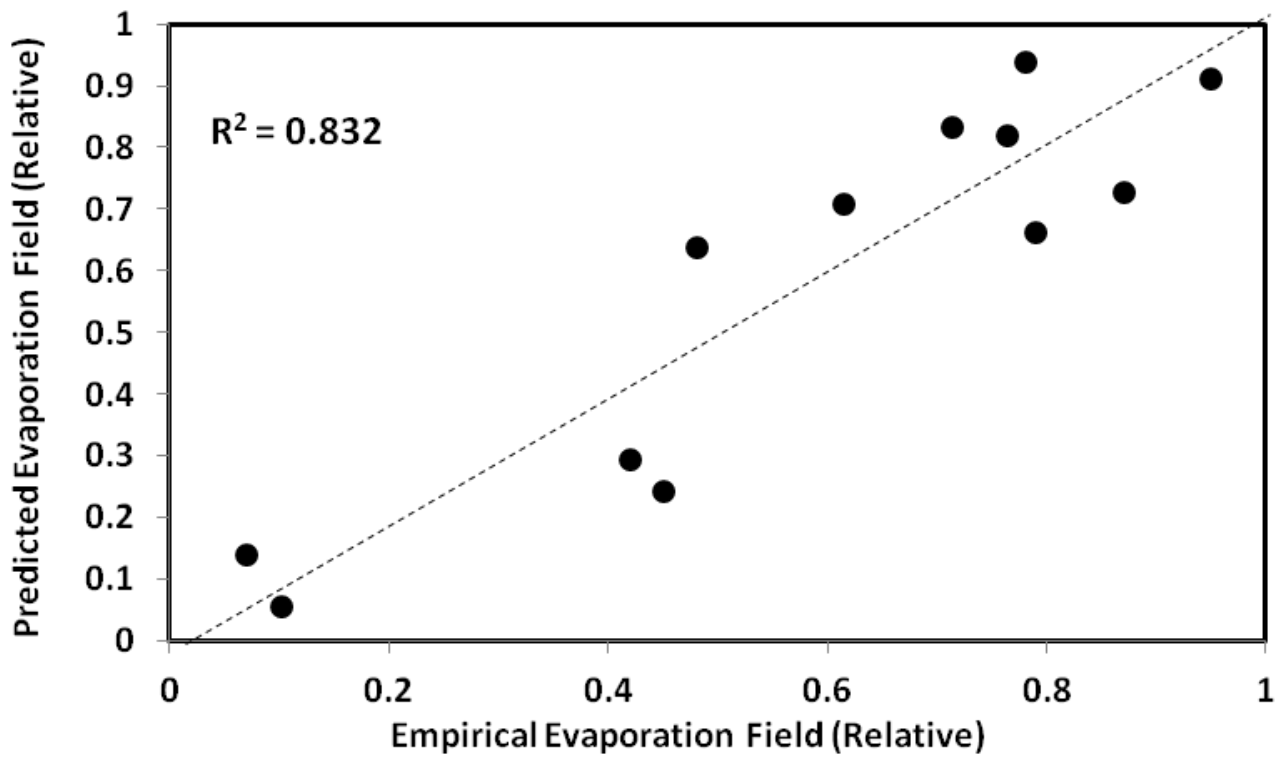

Figure 2. Result of coupled informatics / DFT methodology. As an input of chemistry, we are able to calculate the evaporation field in a high throughput manner. This represents a significant acceleration in the accurate APT reconstruction, while removing user bias. 